\title{
LEPROSY TREATMENT WITH GRASSET'S TUBERCLE ENDOTOXOID
}

\author{
INTERIM REIUR'T
}

\author{
A. R. DAVISON
}

On the supposition that there may be a group antigen for the mycobacterium leprae and the mycobacterium tuberculosis it was decided to try the effect of " Grasset's Tubercle Endotoxoid " on a series of cases of leprosy. This was prepared and presented to us by the South African Institute for Medical Research, Johannesburg.

Clinical results on the treatment of human tuberculosis have been exposed in several papers by E. Grassett:-

I. C. R. Acd. Sci. Paris I935, t.200, I889.

2. C. R. Intern. Congress Pediatrics, Rome, 1937. Acta Paediatr. 22, p. 364 .

3. Tubercule; 1939; vol. 22. p. 397.

4. Proc. Tranv. M. Med. Off. Ass. 1940. vol. 19, p. 239.

Principles and methods of priparation:

According to the method described by E. Grasset (I935; 1939) tubercle endotoxoid consists of the antigenic, water soluble fractions obtained from tubercle bacilli by an extraction process of repeated freezing at low temperature. By submitting the resulting antigen to the detoxicating action of formol at incubator temperature, after the addition of hydrolyzed peptic medium, an atoxic stable vaccine " tubercle endotoxoid " is obtained, which has retained the antigenic properties of the original endotoxin. It can be injected in doses of several c.c. without giving rise to toxic or allergic symptoms in either normal or tuberculous subjects.

The following types of cases were selected for treatment with Grasset's tubercle endotoxoid. Where possible similar cases were selected as controls. They were left on routine anti-leprotic treatment and their condition was noted in November and again in June.

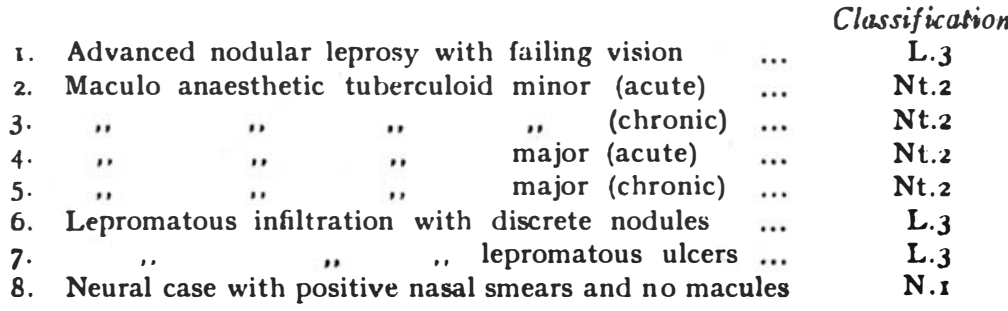


9. Early maculo-anaesthetic with new macules ... ...

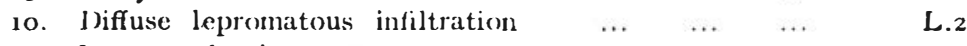

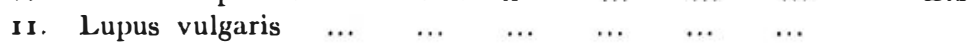

The injections were given subcutaneously bi-weekly commencing at 0.05 c.c., and rising each week until 2.0 c.c. were given at each injection. The cases were treated for 6 months.

Local reactions were experienced in several instances in the first two weeks. These varied from tenderness at the site of the injection to brawny swelling of the arm which passed off in 48 hours. Case No. 2 was not satisfied with his progress and asked to have daily intradermal injections as well.. This was done in I c.c. doses during the month of June, with no obvious beneficial effect.

In November, 1939, each case was Mantoux tested, the sedimentation index and the weight were recorded. A clinical description of the lesions was recorded and the case was photographed. Treatment was commenced in December, 1939.

\section{Case Reconds}

Case 1. Vision very weak, eyes not painful but watery. As a result of iritis and keratitis the oculist reported " Narrow stiff pupil and cataract on right side, vision being $2 / 60$. Fundus cannot be observed any more. His vision will gradually but slowly deteriorate a little more. The left eye is atrophic."

November, 1939: Clinically he was noted to have erythematous nodular infiltration of face and limbs with occasional ulcerating nodules. Back and chest covered by infiltration varying in colour from red to brown.

Sedimentation index 42. Weight 135 lbs. Mantoux positive.

June, 1940: The report was-" Vision weaker. States he eats and sleeps better. Ulcerating nodules generally healed but have recurred on left hand and flank. No change in infiltration.

Sedimentation index 44. Weight 139 lbs.

Conclusion-no improvement.

Case 2. November, 1939 : Clinical condition-dusky heavy spongy maculae on forehead, limbs and abdomen; erythematous and thickened left ear, healing macule back of neck but active (raised and erythematous) where it extends into scalp. Black desquamating border and spreading erythematous margin to macule on buttock.

Sedimentation index 40 . Weight 145 lbs. Mantoux negative.

June, 1940 : Lesions definitely less raised, not spreading, but erythema persists. 
Sedimentation index 2. Weight I45 lbs.

conclusion-slightly improved.

Case" 2 (a) : (Control of Case 2.)

November, 1939: Raised red pea-sized maculae on face, abdomen and back. Flat centres on shoulder.

Sedimentation index 32 . Weight I50 lbs. Mantoux negative.

June, 1940 : No clinical change.

Sedimentation index 3. Weight I45 lbs.

Conclusion-has not responded as well as Case 2.

Case 3. November, 1939: Clinical condition-active maculae with granular crythematous margins and erythema on chest. Isolated patches of residual erythema in sites of old maculae on back and legs.

Sedimentation index I.5. Weight I65 lbs. Mantoux negative.

Iune, 1940 : Internal zone of erythema on chest maculae had faded but margins are still raised and erythematous. Other maculae unchanged.

Sedimentation index I.5. Weight $156 \frac{1}{2}$ lbs. (possibly due to playing football).

Conclusion-slightly improved.

Case 3(a): (Control of Case 3).

November, 1939: Active maculae with raised dusky red margins.

Sedimentation index I.5. Weight I50 lbs. Mantoux negative.

June, 1940 : Maculae active and coalescing.

Sedimentation index 2. Weight $150 \mathrm{lbs}$.

Conclusion-not improved.

Case 4. November, 1939: Face is one erythematous slightly raised blush with suggestion of infiltration but smears show only occasional bacilli. Spongy maculae with silvery desquamation on trunk. Maculae more erythematous but not desquamating on limbs.

Sedimentation index 56. Weight 85 lbs. Mantoux positive.

June, 1940 : Lesions on face completely resolved leaving tissue papery skin. Erythema faded from maculae of trunk and limbs. Lesions now flat.

Sedimentation index 48. Weight $8 \mathrm{I}$ lbs. (plays football). Smears negative.

Conclusion-very marked improvement. 
Case 4(a): (Control of Case 4).

November, 1939: Face is diffusely erythematous around multiple flat dusky healing maculae (tuberculoid major). Small erythematous maculae on back have coalesced into plaque on right flank.

Sedimentation index I.5. Weight I50 lbs. Mantoux negative.

June, 1940 : Infiltration absorbed in right flank. Erythema faded from face. Less erythema in other macule.

Sedimentation index 3. Weight 160 lbs.

Conclusion-improved but not to same extent as Case 4.

Case 5. November, 1939: All maculae on trunk and limbs have raised margins which are blackened and desquamating. There is diffuse erythema in a tuberculoid macule on buttock. Some isolated patches of raised erythema are present in one macule.

Sedimentation index ro. Weight 122 lbs. Mantoux negative.

June, 1940 : Lesions show less elevation of the margins but erythema persists. There has been no spread of the maculae.

Sedimentation index 2. Weight II2 lbs.

Conclusion-slight improvement.

(No similar case available as control.)

Case 6. November, 1939 : Discrete nodules on face project above diffuse infiltration. Ears not pendulous. Diffuse erythematous infiltration on abdomen. Nodules above kidney and on arm are ulcerating. Mottled maculae on chest and back.

Sedimentation index 45. Weight 130 lbs. Mantoux negative.

June, 1940 : Treatment cancelled owing to oedema forearms, hands and feet. Trace albumin in urine. New erythematous maculae slightly raised on chest, abdomen and arms. No change in nodules.

Sedimentation index 45. Weight II8 lbs.

Conclusion-worse.

Case 6(a): (Control of Case 6).

November, 1939 : Diffuse infiltration face with discrete nodules on nose and chin.

Sedimentation index 26. Weight I45 lbs.

June, 1940 : No change clinically.

Sedimentation index 6o. Weight I45 lbs.

Case 7. November, 1939: An advanced lepromatous case showing heavy infiltration of face with ichythotic margins to maculae on trunk. Very weak-has to be carried. Selected to 
see the effects on ulcers of legs which are very deep with grey anaemic sloughs.

Sedimentation index 60. Weight II5 lbs. Mantoux positive.

January, 1940: Ulcers improving-now are red, granulating and closing in from sides.

February, 1940): Ulcers deeper with punched out margins. Granulations pale.

1)arch, 194(): Too ill to disturb.

Conclusion-Case unsuitable for investigation.

Case 8. November, 1939 : An old-standing maculo anaesthetic case. The maculae have faded leaving traces on shoulders and front of thighs. Perforating ulcers both feet. Gynaecomastia. Has been clinically arrested for two years but every routine monthly smear is positive.

Sedimentation index 59. Weight 125 lbs. Mantoux negative.

June, 1940 : Ulcers on foot healed. Routine monthly smears continued positive until February. Scanty bacilli found in March. Negative in April, May and June.

Sedimentation index 42 . Weight 127 lbs.

Conclusion-satisfactory progress but conclusion now would be premature.

Case 8(a): (Control of Case 8).

This case is healed to all clinical appearances, but monthly nasal smears are consistently positive.

Sedimentation index Io. Weight 165 lbs.

June, 1940 : New maculae with pink puffy margins on chest. All smears from November to June have been positive except the one of May month.

Sedimentation index 2. Weight r45 lbs.

Conclusion-not so satisfactory as Case 8 .

Case 9. November, 1939: Maculae on face raised and red with margins which fade into normal skin. Faint erythema on forearms. Maculae are plaque-like around left elbow. Multiple raised new maculae on left buttock. He is husky and wellformed but posture is bad, abdomen protrudes and chest is hollow.

Sedimentation index $\frac{1}{2}$ (Repeated I). Weight I4O lbs. Mantoux negative.

In April developed crepitations of apex and large pleural effusion. Pulmonary tuberculosis was diagnosed despite negative sputum.

June, 1940: Marked improvement in all leprotic lesions which are less erythematous and less raised. This is what we would 
expect in any patient with active tuberculosis 'even without antileprotic treatment.

Sedimentation index 27. Weight 127 lbs. Mantoux negative.

Conclusion-not possible to cvaluate effect of treatment.

Case 9(a): (Control of Case 9).

Novcmber, 1939: Dusky margins with raised erythematous centres to maculae on face. Margins becoming granular but still erythematous on abdomen. Multiple new maculae on back with raised margins.

Sedimentation index 53. Weight 150 lbs. Mantoux positive

June, 194(): The erythema and raised margins have subsided on face. The maculae are flat and inactive on trunk.

Sedimentation index I. Weight 139 lbs.

Conclusion-improvement more marked than in control.

Case 10. November, 1939: Diffuse infiltration face, slightly more thickened over eyebrows. Ears slightly pendulous. Diffuse erythematous infiltration chest and arms, heavier infiltration down spine.

Sedimentation index 40. Weight 135 lbs. Mantoux negative.

June, 1940: No change in clinical condition.

Sedimentation index 6o. Weight 128 lbs.

Conclusion-no improvement.

Case 10(a): (Control of Case Io).

November, 1939: Diffuse infiltration of face with coalescing plaques on trunk and limbs.

Sedimentation index 28. Weight I20 lbs.

June, 194(): Infiltration of face, flanks and forearms worse.

Sedimentation index 38 . Weight I3I lbs.

Conclusion-disease has advanced more rapidly than in control Case ro.

Case 11: This case was admitted in April, 1939, as a certified leprosy patient. On the face and left buttock were identical large lesions. The margins were elevated and described as " blackish fungating irregular edge which bleeds easily". The centres were of healed and glistening scar tissue. There was no anaesthesia of the face and only slight impairment of sensation in one portion of the scar on the buttock. No other nerves were involved. No leprosy bacilli could be found. Cultures on three media, Dorset, Sabourand and Harold were negative. Dr. Muir saw the case in July and agreed that it was probably lupus vulgaris as a 
tuberculoid macule showing such destruction of tissue would certainly have shown more nerve involvement.

In November, 1939, there had been no change in the clinical condition despite anti-leprotic treatment. He was placed on T.B. endotoxoid and the lesions commenced to resolve.

In March, 1940, he was examined by the Annual Leprosy Board and was discharged. The lesion on the face was almost completely flat but on the buttock some clevation of the edge was still present in certain parts.

Conclision-Lupus vulgaris responded to treatment by T.R. endotoxoid.

\section{SUmmary}

1. Ten cases of leprosy and one case of Lupus Vulgaris were trented for six months with Grasset's Tuberculosis Endotoxoid.

2. The Lupus Vulgaris was markedly improved (Case I1).

3. One case (Case 4) of leprosy-an acute tuberculoid major-was markedly improved.

4. Cases 2, 3 and 5 were slightly improved. These were neural cases of the tuberculoid type.

5. Case 8 who had persistently positive nasal smears has now been negative for three months.

6. No beneficial effect was obtained in the cutaneous type (Cases I, 6,7 and 10).

7. Cise 9 developed signs of presumable pulmonary tuberculosis while undergoing the treatment.

8. The greatest improvement was obtained $i_{n}$ a Mantoux positive case.

\section{Discussion}

The improvement obtained in all the neural cases of the tuberculoid type, particularly Case 4, points to the fact that Grasset's endotoxoid has some beneficial effect on tuberculoid lesions. We are satisfied that the improvement in Case 4 is greater than we have previously obtained with any other medicaments. It is our intention to select other cases of this type for further study.

I have to thank the Secretary for Public Health for the Union of South Africa for authority to conduct these experiments at the Pretoria Leper Institution. Also I have to thank Dr. Grasset and the South African Institute for Medical Research for the material as well as the interest and help which was given me. 